\title{
«smarter medicine»: die «Top-5-Liste» der SGN
}

Trägerschaft «smarter medicine»

Die amerikanische Gesellschaft für Nephrologie veröffentlichte im Jahr 2012 fünf Empfehlungen im Rahmen ihrer Initiative «Choosing Wisely». Fasziniert von den beiden Säulen der Kampagne - wertvolle Fürsorge (d.h. mit einem günstigen Verhältnis von Gesamtkosten und Nutzen) und gemeinsame Entscheidungsfindung - hat die Schweizerische Gesellschaft für Nephrologie (SGN) 2016 diese während einer Klausur des SGNAusschusses an den schweizerischen Kontext angepasst und veröffentlicht. Zwei Jahre später stellt sich im Rahmen der Initiative "smarter medicine» die Frage: Ist die Liste noch aktuell? Die Antwort lautet Ja.

Die Schweizerische Gesellschaft für Nephrologie empfiehlt, folgende fünf Interventionen zu vermeiden:

1. Beginnen Sie keine chronische Dialyse ohne Einbeziehung des Patienten und seiner Familie im Entscheidungsprozess

Die Einleitung einer chronischen Dialyse sollte individuell im Rahmen einer Diskussion mit dem Patienten, seiner Familie und den behandelnden Ärzten entschieden werden. Dieser Prozess beinhaltet, dass man die Wünsche und Ziele der einzelnen Patienten erfährt und Informationen zu Prognose und dem zu erwartenden Nutzen oder/und Schaden einer Dialyse im Rahmen dieser Ziele liefert. Begrenzte Beobachtungsdaten bei älteren Patienten mit hoher Komorbidität weisen darauf hin, dass sich das Überleben nicht wesentlich unterscheidet bei einer chronischen Dialyse im Gegensatz zu einer konservativen Behandlung.

2. Führen Sie kein onkologisches Screening für asymptomatische Patienten mit dialysepflichtiger Nierenerkrankung durch, ohne Risiken und Nutzen besprochen zu haben

Wegen der hohen Sterblichkeitsrate bei Patienten mit dialysepflichtiger Nierenerkrankung verbessert ein onkologisches Screening - z.B. Mammographie, Darmspiegelung, Prostata-spezifisches Antigen (PSA) und Pap-Abstrich - das Überleben nicht. Falsch positive Tests können Schaden verursachen: unnötige Prozedu-

\section{Die Kampagne "smarter medicine»}

Der neu gegründeteTrägerverein verleiht der 2014 in der Schweiz lancierten Initiative "smarter medicine" Aufwind: Nebst medizinischen Fach- und Berufsorganisationen unterstützen auch Patienten- und Konsumentenorganisationen die Kampagne. Sie möchten gemeinsam die Öffentlichkeit dafür sensibilisieren, dass bei gewissen Behandlungen weniger Medizin mehr Lebensqualität für die Betroffenen bedeuten kann.

Sie knüpfen an die erfolgreiche amerikanische Initiative "Choosing Wisely» an, welche zum Ziel hat, nicht nur «kluge Entscheidungen» herbeizuführen, sondern auch die offene Diskussion zwischen Ärzteschaft, den Patienten und der Öffentlichkeit zu fördern.

In den nächsten Monaten werden weitere medizinische Fachgesellschaften sogenannte Top-5-Listen mit unnützen Behandlungen in ihrem Fachbereich publizieren. Weitere Informationen zum Trägerverein und eine Übersicht über die bestehenden Top-5-Listen ist unter www.smartermedicine.ch zu finden.

ren, Überbehandlung, fälschlich gestellte Diagnosen und erhöhten Stress. Eine Krebsvorsorge sollte individuell erfolgen unter Einbeziehen der Risikofaktoren, der zu erwartenden Überlebensrate oder im Hinblick auf eine mögliche Nierentransplantation.

3. Vermeiden Sie nicht-steroidale Antirheumatika (NSAR) bei Personen mit Bluthochdruck, Herzversagen und/oder chronischer Nierenerkrankung Der Gebrauch von NSAR, auch von CyclooxygenaseTyp-2(COX-2)-Hemmern, zur pharmakologischen Behandlung von Muskel- und Skelettschmerzen kann den Blutdruck erhöhen, die Wirkung antihypertensiver Mittel mindern, Flüssigkeitsretention verursachen und die Nierenfunktion verschlechtern. Andere Wirkstoffe wie Paracetamol, Tramadol oder der kurzfristige Gebrauch von Opiaten können weniger nephrotoxisch und genauso wirksam wie NSAR sein.

4. Beginnen Sie keine Behandlung mit Erythropoiese-stimulierenden Wirkstoffen (ESA) bei Patienten mit asymptomatischer, chronischer Nierenerkrankung und Hämoglobinspiegeln $\geq 10 \mathrm{~g} / \mathrm{dl}$

Eine Normalisierung der Hämoglobinspiegel durch 


\section{Zur Entstehung dieser Liste}

Die American Society of Nephrology (ASN) führt eine Arbeitsgruppe für Qualität und Patientensicherheit. Diese fördert das Engagement der ASN, Patienten eine qualitativ hochwertige Versorgung zu bieten und das Bewusstsein für Fragen der Patientensicherheit aller Fachkräfte zu schärfen, die Patienten mit Nierenerkrankungen betreuen. Die Gremien der ASN brachten Expertisen in die Arbeitsgruppe ein, um sicherzustellen, dass alle Bereiche der Nephrologie enthalten sind. Die endgültige Liste der fünf Punkte wurde einstimmig von der ASN verabschiedet und 2012 im Rahmen der Initiative "Choosing Wisely" veröffentlicht.

Die Schweizerische Gesellschaft für Nephrologie hat diese Liste während einer Klausur des SGN-Ausschusses an den schweizerischen Kontext angepasst, im Mai 2016 veröffentlicht und nun unter das Dach «smarter medicine» gestellt. Eine ausführliche Literaturliste sowie Empfehlungen von weiteren medizinischen Fachgesellschaften sind unter www.smartermedicine.ch abrufbar.

\section{Korrespondenz:}

Trägerverein

smarter medicine c/o SGAIM

Monbijoustrasse 43

CH-3001 Bern

smartermedicine[at]sgaim.ch

ESA-Gabe bei Patienten mit chronischer Nierenerkrankung konnte weder einen Überlebensvorteil zeigen noch die die kardiovaskuläre Morbidität senken und ist im Vergleich zu einem moderaten Hämoglobinzielspiegel von 9-11 g/dl sogar schädlich. ESA sollten verschrieben werden, um Transfusionen zu minimieren. Der optimale Hämoglobinzielspiegel ist der tiefstmögliche Wert unter Berücksichtigung der individuellen Patientenbedürfnisse.
5. Vermeiden Sie, wenn möglich, Venenkatheter am Arm bei Patienten mit chronischer Nierenfunktionseinschränkung Stadium 4-5, wenn eine Hämodialyse geplant ist

Die Erhaltung der Venen ist wichtig für Patienten mit chronischer Nierenfunktionseinschränkung Stadium 4-5. Arteriovenöse Fisteln (AVF) sind im Vergleich zu zentralen Venenkathetern und Kunststoffinterponaten der beste Hämodialysezugang mit weniger Komplikationen und niedrigerer Sterberate. Häufige Venenpunktionen sind schädlich und zerstören mögliche AVF-Stellen. Periphere und zentrale Venenkatheter können Thrombosen und Stenosen auslösen. Eine frühe nephrologische Mitbetreuung kann unnötige Venenpunktionen und -katheter vermeiden helfen und erhöht den Gebrauch von AVF als primären Hämodialysezugang.
Diese Empfehlungen, nun unter dem Dach «smarter medicine» veröffentlicht, erinnern daran, dass der Wert jeder diagnostischen oder therapeutischen Handlung nur anhand individueller Faktoren beurteilt werden kann und dass sie die direkte Beteiligung des Patienten und seiner Umgebung erfordert. 